T.H. KIM

KODAI MATH. J.

15 (1992), 296-309

\title{
NONLINEAR ERGODIC THEOREMS OF ALMOST-ORBITS OF NON-LIPSCHITZIAN SEMIGROUPS
}

\author{
TAE HWA Kim
}

\begin{abstract}
In this paper, we shall establish the weak convergence and nonlinear ergodic theorems for reversible semigroups of weakly asymptotically nonexpansive type in Banach spaces.
\end{abstract}

\section{Introduction}

Let $G$ be a semitopological semigroup, i. e., $G$ is a semigroup with a Hausdorff topology such that for each $a \in G$ the mappings $s \mapsto a \cdot s$ and $s \rightarrow s \cdot a$ from $G$ to $G$ are continuous. $G$ is called right reversible if any two closed left ideals of $G$ have nonvoid intersection. In this case, $(G, \geqslant)$ is a directed system when the binary relation " $\succcurlyeq$ " on $G$ is defined by

$$
t \succcurlyeq s \quad \text { if and only if }\{s\} \cup \overline{G s} \supseteqq\{t\} \cup \overline{G t}, \quad s, t \in G \text {. }
$$

Right reversible semitopological semigroups include all commutative semigroups and all semitopological semigroups which are right amenable as discrete semigroups (see [7, p. 335]). Left reversibility of $G$ is defined similarly. $G$ is called reversible if it is both left and right reversible.

Let $G$ be a semitopological semigroup with a binary relation " $\succcurlyeq$ " which directs $G$. Let $C$ be a nonempty closed convex subset of a real Banach space $E$ and let a family $\Im=\{S(t): t \in G\}$ be a (continuous) representation of $G$ as continuous mappings on $C$ into $C$, i.e., $S(t s) x=S(t) S(s) x$ for all $t, s \in G$ and $x \in C$, and for every $x \in C$, the mapping $t \mapsto S(t) x$ from $G$ into $C$ is continuous. A representation $\Im=\{S(t): t \in G\}$ of $G$ on $C$ is called reversible [resp., right (left) reversible] if $G$ is reversible [resp., right (left) reversible]. In this paper, we also consider a non-Lipschitzian semigroup of mappings: a representation $\mathfrak{\Im}=\{S(t): t \in G\}$ of $G$ on $C$ is said to be a semigroup of weakly asymptotically

*Supported by NON DIRECTED RESEARCH FUND, Korea Research Foundation, 1990. AMS Subject Classification (1980): 47H10

Keywords and phrases. Weak convergence, nonlinear ergodic theorem, almost-orbit, weakly asymptotically nonexpansive type, asymptotic center.

Received May 20, 1991 ; January 14, 1992. 
nonexpansive type (simply, w.a.n.t.) on $C$ if, for each $x \in C$ and each bounded subset $D$ of $C$,

$$
\lim \sup _{t} \sup _{y \in D}(\|S(t) x-S(t) y\|-\|x-y\|) \leqq 0 .
$$

Immediately, we can see that the semigroups of w.a.n.t. include all semigroups of nonexpansive mappings with directed systems. In particular, if $\Im=\{S(t)$ : $t \in G\}$ is a Lipschitzian representation of $G$ with an additional condition, i. e., $\lim _{t} \sup k_{t} \leqq 1$ (see [15]), then it is obviously of w.a.n.t. In cases where $G=N$, $S=S(1)$, we have $S(n)=S^{n}$ for each $n \in N$, where $N$ denotes the set of natural numbers. Then, when the semigroup $\Im=\{S(n): n \in N\}$ is of w. a.n.t., $S: C \rightarrow C$ is simply said to be a mapping of w.a.n.t. For a mapping $S: C \rightarrow C$ of a.n.t., see Kirk [9]. And we say that a function $u: G \rightarrow C$ is an almost-orbit of $\Im=\{S(t): t \in G\}$ (see [13], [15]) if $G$ is right reversible and

$$
\lim _{t}\left(\sup _{s \in G}\|u(s t)-S(s) u(t)\|\right)=0 .
$$

In [15], Takahashi-Zhang established the weak convergence of an almostorbit of a noncommutative Lipschitzian semigroup in a Banach space. In [11], Lau-Takahashi also proved the nonlinear ergodic theorems for a noncommutative nonexpansive semigroup in the space. In this paper, we first shall establish a fixed point theorem for an almost-orbit $\{u(t): t \in G\}$ of the reversible semigroup $\Im=\{S(t): t \in G\}$ of w.a.n.t. in a uniformly convex Banach space, which generalizes the commutative version due to Kiang-Tan [8], and also prove the equivalent conditions of weak convergence of the almost-orbit $\{u(t): t \in G\}$ of $\Im=\{S(t): t \in G\}$, which extend the results due to Miyadera [12] and Emmanule [3, Theorem 2]. Next, we shall carry over the weak convergence and nonlinear ergodic theorems due to Lau-Takahashi [11], Takahashi-Zhang [15] to those for the right reversible semigroup $\Im=\{S(t): t \in G$ of w. a. n.t. Our proofs employ the methods of [8], [11], [12], and [15].

\section{Fixed Point Theorem}

Let $C$ be a nonempty closed convex subset of a real Banach space $E$ and let $G$ be a semitopological semigroup with a binary relation " $\succcurlyeq$ " which directs G.

Definition 2.1. A family $\mathfrak{S}=\{S(t): t \in G\}$ of continuous mappings from $C$ into itself is said to be $a$ (continuous) representation of $G$ on $C$ if $\Im$ satisfies the following:

(a) $S(t s) x=S(t) S(s) x$ for all $t, s \in G$ and $x \in C$;

(b) for every $x \in C$, the mapping $t \mapsto S(t) x$ from $G$ into $C$ is continuous.

Definition 2.2. Let $\Im=\{S(t): t \in G\}$ be a representation of $G$ on $C$. $\Im$ is said to be a semigroup of weakly asymptotically nonexpansive type (simply, 
w.a.n.t.) on $C$ if, for each $x \in C$ and each bounded subset $D$ of $C$,

$$
\inf _{s \in G} \sup _{t \geq s} \sup _{y \in D}(\|S(t) x-S(t) y\|-\|x-y\|) \leqq 0 .
$$

Immediately, we can see that the semigroups of w.a.n.t. indude all semigroups of nonexpansive mappings with directed systems. In particular, if $\Im=$ $\{S(t): t \in G\}$ is a Lipschitzian representation of $G$ with an additional condition, i. e., $\lim \sup _{t} k_{t} \leqq 1$ (see [15]), then it is obviously of w.a.n.t. In cases where $G=N, S=S(1)$, we have $S(n)=S^{n}$ for each $n \in N$, where $N$ denotes the set of natural numbers. Then, when the semigroup $\mathfrak{\Im}=\{S(n): n \in N\}$ is of w. a. n.t., $S: C \rightarrow C$ is simply said to be a mapping of w.a.n.t. For a mapping $S: C \rightarrow C$ of a.n.t., see Kirk [9].

DEFINITION 2.3. Let $G$ be right reversible and let $\Im=\{S(t): t \in G\}$ be as in Definition 2.1. A function $u: G \rightarrow C$ is called an almost-orbit of $\Im=\{S(t): t \in G\}$ (see [13], [15]) if

$$
\lim _{t}\left(\sup _{s \in G}\|u(s t)-S(s) u(t)\|\right)=0 .
$$

Now let $\left\{x_{\alpha}\right\}$ be a bounded net in $C$. Then we define

$$
\begin{gathered}
r\left(x,\left\{x_{\alpha}\right\}\right)=\limsup _{\alpha}\left\|x-x_{\alpha}\right\|, \\
r=r\left(C,\left\{x_{\alpha}\right\}\right)=\inf \left\{r\left(x,\left\{x_{\alpha}\right\}\right): x \in C\right\},
\end{gathered}
$$

and

$$
A\left(C,\left\{x_{\alpha}\right\}\right)=\left\{z \in C: r\left(z,\left\{x_{\alpha}\right\}\right)=r\left(C,\left\{x_{\alpha}\right\}\right)\right\} .
$$

Then, any element of $A\left(C,\left\{x_{\alpha}\right\}\right)$ is called to be an asymptotic center of the net $\left\{x_{\alpha}\right\}$ in $C$. It is well known that if $C$ is weakly compact and convex, then $A\left(C,\left\{x_{\alpha}\right\}\right) \neq \emptyset$ and if $E$ is uniformly convex, then $A\left(C,\left\{x_{\alpha}\right\}\right)$ is a singleton set and it will be simply denoted by the unique element; see [4].

From now on, unless other specified, let $G, E, C$, and $\Im=\{S(t): t \in G\}$ be as in Definition 2.2 and $F(\Im)$ denote the set of all common fixed points of $\Im=$ $\{S(t): t \in G\}$ in $C$, i. e., $F(\Im)=\{x \in C: S(t) x=x$ for all $t \in G\}$. We begin with the following:

LEMMA 2.4. Let $G$ be right reversible and let $E$ be uniformly convex. If $\{u(t): t \in G\}$ is a bounded almost-orbit of $\Im=\{S(t): t \in G\}$ and $r=r(C,\{u(t)\})=0$, then $\lim _{t} S(s) u(t)=c \in F(\Im)$ for each $s \in G$, where $c=A(C,\{u(t)\})$.

Proof. Since $r=r(c,\{u(t)\})=0$, clearly $c=\lim _{t} u(t)=\lim _{t} u(s t)$ for each $s \in G$. Let $s, t \in G$. Then we obtain

$$
\begin{aligned}
\|c-S(s) u(t)\| & \leqq\|c-u(s t)\|+\|u(s t)-S(s) u(t)\| \\
& \leqq\|c-u(s t)\|+\phi(t)
\end{aligned}
$$


where $\phi(t)=\sup _{s \in G}\|u(s t)-S(s) u(t)\|$. Since $\{u(t): t \in G\}$ is an almost-orbit of $\Im=\{S(t): t \in G\}, \lim _{t} \phi(t)=0$, and now taking $\lim _{t}$ sup in both sides, we obtain $\lim _{t} S(s) u(t)=c$, and hence $S(s) c=S(s)\left(\lim _{t} u(t)\right)=\lim _{t} S(s) u(t)=c$, i. e., $\quad c \in F(\mathfrak{S})$. This completes the proof.

We say that $\Im=\{S(t): t \in G\}$ is proximately nonexpansive (see [8] if for every $x \in C$, and every $\beta>0$, there exists $t_{0} \in G$ such that $\|S(t) x-S(t) y\| \leqq$ $(1+\beta)\|x-y\|$ for all $t \geqslant t_{0}$, and for any $y \in C$. If $\Im=\{S(t): t \in G\}$ is proximately nonexpansive, then it is of w.a.n.t. Indeed, let $x \in C$ and $D$ a bounded subset of $C$. Then, for given $\varepsilon>0$, take $\beta=\frac{\varepsilon}{M}$, where $M=\sup _{y \in D}\|x-y\|$.

In order to measure the degree of strict convexity (rotundity) of $E$, we define its modulus of convexity $\delta:[0,2] \rightarrow[0,1]$ by

$$
\delta(\varepsilon)=\inf \left\{1-\frac{1}{2}\|x+y\|:\|x\| \leqq 1,\|y\| \leqq 1, \text { and }\|x-y\| \geqq \varepsilon\right\} .
$$

The characteristic of convexity $\varepsilon_{0}$ of $E$ is also defined by

$$
\varepsilon_{0}=\varepsilon_{0}(E)=\sup \{\varepsilon: \delta(\varepsilon)=0\} .
$$

It is well-known (see [4]) that the modulus of convexity $\delta$ satisfies the following properties:

$$
\left\{\begin{array}{l}
\text { (a) } \delta \text { is increasing on }[0,2] \text {, and moreover strictly increasing on }\left[\varepsilon_{0}, 2\right] ; \\
\text { (b) } \delta \text { is continuous on }[0,2) \text { (but not necessarily at } \varepsilon=2) ; \\
\text { (c) } \delta(2)=1 \text { if and only if } E \text { is strictly convex; } \\
\text { (d) } \delta(0)=0 \text { and } \lim _{\varepsilon \rightarrow 2-} \delta(\varepsilon)=1-\frac{1}{2} \varepsilon_{0} ; \\
\text { (e) }\|a-x\| \leqq r,\|a-y\| \leqq r \text { and }\|x-y\| \geqq \varepsilon \\
\Longrightarrow\left\|a-\frac{1}{2}(x+y)\right\| \leqq r(1-\delta(\varepsilon / r))
\end{array}\right.
$$

A Banach space $E$ is said to be unformly convex if $\delta(\varepsilon)>0$ for all positive $\varepsilon$; equivalently $\varepsilon_{0}=0$. Obviously, any uniformly convex space is both strictly convex and reflexive. By properties above, we can see that if $E$ is uniformly convex, then $\delta$ is strictly increasing and continuous on $[0,2]$ (see also [1]).

Now we can present a fixed point thorem for an almost-orbit $\{u(t): t \in G\}$ of the reversible semigroup $\Im=\{S(t): t \in G)$ of w.a.n.t. in a uniformly convex Banach space, which generalizes the commutative version due to Kiang-Tan [8; Theorem 1].

THEOREM 2.5. Let $G$ be reversible and let $E$ be uniformly convex. If an almost-orbit $\{u(t): t \in G\}$ of $\Im=\{S(t): t \in G\}$ is bounded, then $A(C,\{u(t)\})=c \in F(\Im)$. 
Proof. If $r=r(C,\{u(t)\})=0$, the result follows from Lemma 2.4. Assume $r>0$ and we set

$$
d(c)=\lim _{s} \sup \|c-S(s) c\| .
$$

Then, $d(c)<\infty$. In fact, since $\Im=\{S(t): t \in G\}$ is of w.a.n.t., for $c \in C$ and $D=\{u(t): t \in G\}$,

$$
\lim \sup _{t} \sup _{y \in D}(\|S(t) c-S(t) y\|-\|c-y\|) \leqq 0 .
$$

For $s, t \in G$, we have

$$
\begin{aligned}
& \|c-S(t) c\| \leqq\|c-u(t s)\|+\| u(t s)-S(t(u(s)\|+\| S(t) u(s)-S(t) c \| \\
\leqq & \|c-u(t s)\|+\phi(s)+\|S(t) c-S(t) u(s)\| \\
\leqq & 2(\|c\|+M)+\phi(s)-\sup _{y \in D}(\|S(t) c-S(t) y\|-\|c-y\|),
\end{aligned}
$$

where $\quad M=\sup _{t \in G}\|u(t)\|<\infty$ and $\phi(s)=\sup _{t \in G}\|u(t s)-S(t) u(s)\|$. Since $\lim _{s} \phi(s)=0$, taking $\lim _{t}$ sup at first and next $\lim _{s}$ sup in both sides, we obtain

$$
d(c) \leqq 2(\|c\|+M)<\infty .
$$

If $d(c)=0$, then, since $G$ is right reversible, we obtain $S(t) c=\lim _{s} S(t) S(s) c=$ $\lim _{s} S(t s) c=\lim _{s} S(s) c=c$ for each $t \in G$, i. e., $c \in F(\Im)$. Hence, it suffices to show that $d(c)=0$. If not, let $\varepsilon$ be such that $0<\varepsilon<d(c)$. Then, for each $\alpha \in G$, there is $s_{\alpha} \in G$ such that $s_{\alpha} \succcurlyeq \alpha$ and

$$
\left\|c-S\left(s_{\alpha}\right) c\right\|>\varepsilon
$$

Since $E$ is uniformly convex, choose $d>0$ so small that

$$
(r+d)\left[1-\delta\left(\frac{\varepsilon}{r+d}\right)\right]<r,
$$

where $\delta$ is the modulus of convexity of $E$. For this $d>0$, by (2.1) with $c \in C$ and $D=\{u(s): s \in G\},(2.2)$, and (2.3), there exists $\alpha \in G$ such that

$$
\begin{gathered}
\|S(t) c-S(t) y\| \leqq\|c-y\|+\frac{d}{3}, \\
\sup _{s \in G}\|u(s t)-S(s) u(t)\|<\frac{d}{3},
\end{gathered}
$$

and

$$
\|c-u(t)\|<r+\frac{d}{3}
$$

for all $t \geqslant \alpha$ and all $y \in D$. Then, for this $\alpha$, we can also choose $s_{\alpha} \in G$ with $s_{\alpha} \succcurlyeq \alpha$ and 


$$
\left\|c-S\left(s_{\alpha}\right) c\right\|>\varepsilon
$$

Then, for all $t \succcurlyeq \alpha$, we have

$$
\begin{aligned}
& \left\|u\left(s_{\alpha} t\right)-S\left(s_{\alpha}\right) c\right\| \\
\leqq & \left\|u\left(s_{\alpha} t\right)-S\left(s_{\alpha}\right) u(t)\right\|+\left\|S\left(s_{\alpha}\right) u(t)-S\left(s_{\alpha}\right) c\right\| \\
< & \frac{d}{3}+\|u(t)-c\|+\frac{d}{3}<r+d .
\end{aligned}
$$

Let $b_{0}=s_{\alpha} \alpha$ and $t \succcurlyeq b_{0}$. Then, since $G$ is left reversible, we obtain

$$
\sup _{t \geq b_{0}}\left\|u(t)-S\left(s_{\alpha}\right) c\right\|=\sup _{t \geq a}\left\|u\left(s_{\alpha} t\right)-S\left(s_{\alpha}\right) c\right\|<r+d .
$$

Clearly, we obtain

$$
\sup _{t \geq b_{0}}\|c-u(t)\|<r+d .
$$

By the definition of $\delta$ (see (e) of (2.6)), it follows that

$$
\sup _{t \geq b_{0}}\left\|u(t)-\frac{1}{2}\left(c+S\left(s_{\alpha}\right) c\right)\right\| \leqq(r+\sigma)\left[1-\delta\left(\frac{\varepsilon}{r+\sigma}\right)\right] .
$$

Setting $z=\frac{1}{2}\left(c+S\left(s_{a}\right) c\right) \in C$, it implies that

$$
\begin{aligned}
r & \leqq r(z,\{u(t)\}) \leqq \sup _{t \geq b_{0}}\|\mid u(t)-z\| \\
& \leqq(r+\sigma)\left[1-\delta\left(\frac{\varepsilon}{r+\sigma}\right)\right]<r,
\end{aligned}
$$

which gives a contradiction. The proof is completed.

\section{Weak Convergence}

In this section, we present the weak convergence of almost-orbits $\{u(t) \mid t \in G\}$ of the reversible semigroup $\mathfrak{\Im}=\{S(t): t \in G\}$ of w.a.n.t. For a function $u: G \rightarrow C$, let $\omega_{w}(u)$ denote the set of all weak limits of subnets of the net $\{u(t): t \in G\}$ and we set

$$
E(u)=\{y \in C: \lim \|u(t)-y\| \text { exists }\} .
$$

Then, the following lemma is crucial for our arguement:

LEMMA 3.1. Let $G$ be right reversible and let $\{u(t): t \in G\},\{v(t): t \in G\}$ almost-orbits of $\mathfrak{J}=\{S(t): t \in G\}$. Then the limit of $\|u(t)-v(t)\|$ exists. In particular, $F(\mathfrak{\Im}) \leqq E(u)$.

Proof. Since $\Im=\{S(t): t \in G\}$ is of w.a.n.t., it follows that, for each 
$s \in G$ and $D=\{v(s)\}$,

$$
\lim _{t} \sup (\|S(t) u(s)-S(t) v(s)\|-\|u(s)-v(s)\|) \leqq 0,
$$

Therefore, for each $s, t \in G$,

$$
\begin{aligned}
\|u(t s)-v(t s)\| & \leqq\|u(t s)-S(t) u(s)\|+\|S(t) u(s)-S(t) v(s)\|+\|S(t) v(s)\|-v(t s) \| \\
& \leqq \phi(s)+(\|S(t) u(s)-S(t) v(s)\|-\|u(s)-v(s)\|)+\|u(s)-v(s)\|+\phi(s),
\end{aligned}
$$

where $\phi(s)=\sup _{t}\|u(t s)-S(t) u(s)\|, \quad \phi(s)=\sup _{t}\|v(t s)-S(t) v(s)\|$. At first, taking $\lim _{t}$ sup at both sides, we get

$$
\lim _{t} \sup \|u(t)-v(t)\| \leqq \lim _{t} \sup _{t}\|u(t s)-v(t s)\| \leqq \phi(s)+\phi(s)+\|u(s)-v(s)\| .
$$

Since $\{u(t): t \in G\},\{v(t): t \in G\}$ are almost-orbits of $\Im=\{S(t): t \in G\}, \lim _{s} \phi(s)=0$ and $\lim _{s} \phi(s)=0$, and now taking $\lim _{s} \inf$ in both sides, $\lim _{t}\|u(t)-v(t)\|$ exists. Now let $z \in F(\Im)$ and put $v(t) \equiv z$ for all $t \in G$. Then $\{v(t): t \in G\}$ is an almostorbit and hence the limit of $\|u(t)-z\|$ exists, i.e., $z \in E(u)$.

When $\left\{x_{\alpha}\right\}$ is a net in a Banach space $E$ and $x \in E, x_{\alpha} \rightarrow x\left(x_{\alpha} \rightarrow x\right)$ means the strong (weak) convergence to $x$ of the net $\left\{x_{\alpha}\right\}$, respectively.

Recall that a Banach space $E$ is said to satisfy Opial's condition if, for any net $\left\{x_{\alpha}\right\}$ in $E$ with $x_{\alpha} \rightarrow x \in E$,

$$
\limsup _{\alpha}\left\|x_{\alpha}-x\right\|<\lim _{\alpha} \sup \left\|x_{\alpha}-y\right\|, \quad \forall y(\neq x) \in E .
$$

(see [10, Lemma 2.1]). For any sequence in $E$, see [14, Lemma 1]. For more details, see also [3] and [5]. We are now ready to prove the equivalent conditions of weak convergence for the almost-orbit $\{u(t): t \in G\}$ of the reversible semigroup $\Im=\{S(t): t \in G\}$ of w.a.n.t., which extends the results due to Miyadera [12] and Emmanuele [3, Theorem 2].

THEOREM 3.2. Let $G$ be reversible and let $E$ be uniformly convex with Opial's condition. Let $u=\{u(t): i \in G\}$ be an almost-orbit of $\Im=\{S(t): t \in G\}$. Then the following conditions are equivalent

(i) $w-\lim _{t} u(t)$ exists;

(ii) $F(\Im) \neq \emptyset$ and $\omega_{w}(u) \subseteq F(\Im)$;

(iii) $E(u) \neq \emptyset$ and $\omega_{w}(u) \leqq E(u)$.

Proof. Since $E$ is uniformly convex and $\{u(t): t \in G\}$ is bounded in $C$ by (i), by Theorem 2.5., there exists a unique asymptotic center $A(C,\{u(t)\})=$ $c \in F(\Im)$. Then, Opial's condition also gives that $c=w-\lim _{t} u(t)$. Hence we have $\omega_{w}(u)=\{c\} \subseteq F(\Im)$. Thus (i) implies (ii). By Lemma 3.1., clearly (ii) implies (iii). Also to show that (iii) implies (i), apply the method of Theorem 1 due to Emmanuele [3]. 
LEMMA 3.3. Let $G$ be right reversible and let $E$ be uniformly convex. Let $u=\{u(t): t \in G\}$ be an almost-orbit of $\mathfrak{\Im}=\{S(t): t \in G\}$. Suppose $F(\mathfrak{\Im}) \neq \emptyset$ and let $y \in F(\Im)$ and $0<\alpha \leqq \beta<1$. Then, for any $\varepsilon>0$, there is $t_{0} \in G$ such that

$$
\| S(t)(\lambda u(s)+(1-\lambda) y)-(\lambda S(t) u(s)+(1-\lambda) y \|<\varepsilon
$$

for all $t, s \succcurlyeq t_{0}$ and $\lambda \in[\alpha, \beta]$.

Proof. Since $F(\Im) \neq \emptyset$, we may assume that $\{u(t): t \in G\}$ is bounded, and hence $D=\{\lambda u(t)+(1-\lambda) y: t \in G, 0 \leqq \lambda \leqq 1\}$ is bounded. Let $\varepsilon>0$ and let $r=\lim _{t}\|u(t)-y\|$ by Lemma 3.1. If $r=0$, since $\Im=\{S(t): t \in G\}$ is of w.a.n.t. on $C$, for $y \in C$ and $D$, there exists $t_{0} \in G$ such that

$$
\|y-S(t) z\|<\|y-z\|+\frac{\varepsilon}{4}
$$

and

$$
\|u(t)-y\|<\frac{\varepsilon}{4} \text { for } t \geqslant t_{0} \text { and } z \in D .
$$

Hence, for $s, t \succcurlyeq t_{0}$ and $0 \leqq \lambda \leqq 1$, we have

$$
\begin{aligned}
& \|S(t)(\lambda u(s)+(1-\lambda) y)-(\lambda S(t) u(s)+(1-\lambda) y)\| \\
\leqq & \|S(t)(\lambda u(s)+(1-\lambda) y)-y\|+\lambda\|S(t) u(s)-y\| \\
\leqq & 2\left(\lambda\|u(s)-y\|+\frac{\varepsilon}{4}\right)<\varepsilon .
\end{aligned}
$$

Now, let $r>0$. Then we can choose $d>0$ so small that

$$
(r+d)\left[1-c \delta\left(\frac{\varepsilon}{r+d}\right)\right]=r_{0}<r .
$$

where $\delta$ is the modulus of convexity of $E$ and $c=\min \{2 \lambda(1-\lambda): \alpha \leqq \lambda \leqq \beta\}$. For $k>0$ with $k<\min \left\{d / 2,\left(r-r_{0}\right) / 2\right\}$, as the above, there exists $t_{0} \in G$ such that

$$
\begin{aligned}
& r-a<\|u(t)-y\|<r+k, \\
& \|y-S(t) z\|<\|y-z\|+\frac{c}{4} d, \quad \text { for } t \geqslant t_{0}, z \in D,
\end{aligned}
$$

and

Suppose that

$$
\|u(t s)-S(t) u(s)\|<a, \quad \text { for } s \geqslant t_{0}, t \in G .
$$

$$
\|S(t)(\lambda u(s)+(1-\lambda) y)-(\lambda S(t) u(s)+(1-\lambda) y)\| \geqq \varepsilon
$$

for some $s, t \geqslant t_{0}$ and $\lambda \in[\alpha, \beta]$. Put $z=\lambda u(s)+(1-\lambda) y, u=(1-\lambda)(S(t) z-y)$ and $v=\lambda(S(t) u(s)-S(t) z)$. Then, it follows that

$$
\|u\| \leqq(1-\lambda)\left(\|y-z\|+\frac{c}{4} d\right)
$$


and

$$
\begin{aligned}
& =(1-\lambda)\left(\lambda\|u(s)-y\|+\frac{c}{4} d\right) \\
& <(1-\lambda)\left(\lambda\left(r+\frac{d}{2}\right)+\frac{c}{4} d\right) \\
& <\lambda(1-\lambda)(r+d)
\end{aligned}
$$

We also have that

$$
\|v\|<\lambda(1-\lambda)(r+d) \text {. }
$$

$$
\|u-v\|=\|S(t) z-(\lambda S(t) u(s)+(1-\lambda) y)\| \geqq \varepsilon
$$

and $\lambda u+(1-\lambda) v=\lambda(1-\lambda)(S(t) u(s)-y)$. By lemma in [6], we have

$$
\begin{aligned}
\lambda(1-\lambda)\|S(t) u(s)-y\| & =\|\lambda u+(1-\lambda) v\| \\
& \leqq \lambda(1-\lambda)(r+d)\left[1-2 \lambda(1-\lambda) \delta\left(\frac{\varepsilon}{r+d}\right)\right] \\
& \leqq \lambda(1-\lambda) r_{0},
\end{aligned}
$$

and hence $\|S(t) u(s)-y\| \leqq r_{0}$. Thus it follows that

$$
\begin{aligned}
\|u(t s)-y\| & \leqq\|u(t s)-S(t) u(s)\|+\|S(t) u(s)-y\| \\
& <a+r_{0}<r-a,
\end{aligned}
$$

which gives a contradiction and the proof is complete.

For $x, y \in E$, we denote by $[x, y]$ the set $\{\lambda x+(1-\lambda) y: 0 \leqq \lambda \leqq 1\}$. For $D \subset E, \overline{c o} D$ denotes the closed convex hull of $D$. The following lemma was proved by Lau-Takahashi [11, Lemma 3].

LEMMA 3.4. Let $E$ be uniformly convex with a Fréchet differentiable norm and let $\left\{x_{\alpha}\right\}$ be a bounded net in $C$. Let $z \in \bigcap_{\beta} \overline{c o}\left\{x_{\alpha}: \alpha \geqslant \beta\right\}, y \in C$ and $\left\{y_{\alpha}\right\} a$ net of elements in $C$ with $y_{\alpha} \in\left[y, x_{\alpha}\right]$ and

$$
\left\|y_{\alpha}-z\right\|=\min \left\{\|u-z\|: u \in\left[y, x_{\alpha}\right]\right\} \text {. }
$$

If $y_{\alpha} \rightarrow y$, then $y=z$.

By using Lemma 3.3 and Lemma 3.4, we obtain the similar result as Theorem 2 in [15] for an almost-orbit $\{u(t): t \in G\}$ of the right reversible semigroup $\Im=\{S(t): t \in G\}$ of w.a.n.t. on $C$ in a uniformly convex Banach space $E$ with a Fréchet differentiable norm.

THEOREM 3.5. Let $G$ be right reversible and let $E$ be uniformly convex with a Fréchet differentiable norm. Suppose that $u=\{u(t): t \in G\}$ is an almost-orbit of $\Im=\{S(t): t \in G\}$ and $F(\Im) \neq \emptyset$. Then the set $\bigcap_{s \in G} \overline{c o}\{u(t): t \geqslant s\} \cap F(\Im)$ consists of at most one point. 
Proof. Since $F(\Im) \neq \emptyset$, we may assume that $\{u(t): t \in G\}$ is bounded. Let $W(u)=\bigcap_{s \in G} \overline{c o}\{u(t): t \succcurlyeq s\}$. Suppose that $x, y \in W(u) \cap F(\Im)$ and $x \neq y$. Put $z=(x+y) / 2$ and $r=\lim _{s}\|u(s)-y\|$ by Lemma 3.1. Since $z \in W(u)$, we have $\|z-y\| \leqq r$. For each $s \in G$, choose $z(s) \in[u(s), z]$ such that

$$
\|z(s)-y\|=\min \{\|v-y\|: v \in[u(s), z]\} .
$$

By the definition of $z(s)$, we have $\|z(s)-y\| \leqq\left\|\frac{z(s)+z}{2}-y\right\| \leqq\|z-y\|$ for all $s \in G$. Therefore, if $\liminf _{s}\|z(s)-y\|=\|z-y\|$, then $\{z(s)\}$ converges strongly to $z$. Otherwise, there exists some $\varepsilon>0$ and $s_{\alpha} \in G$ such that $s_{\alpha} \geqslant \alpha$ and $\left\|z\left(s_{\alpha}\right)-z\right\|>\varepsilon$, for every $\alpha \in G$. Then, by the definition of $\delta$ (see (e) of (2.6)), we have

$$
\left\|\frac{1}{2}\left(z\left(s_{\alpha}\right)+z\right)-y\right\| \leqq\|z-y\| \cdot\left[1-\delta\left(\frac{\varepsilon}{\|z-y\|}\right)\right]
$$

for every $\alpha$. It follows from the definition of $z\left(s_{\alpha}\right)$ and the uniform convexity of $E$ that

$$
\begin{aligned}
& \liminf _{s}\|z(s)-y\| \leqq \lim _{\alpha} \sup \left\|z\left(s_{\alpha}\right)-y\right\| \\
& \leqq\|z-y\| \cdot[1-\delta(\varepsilon /\|z-y\|)]<\|z-y\|,
\end{aligned}
$$

which contradicts the assumption. So, $\lim _{s} z(s)=z$. Therefore, by Lemma 3.4, we obtain $z=y$ and this contradicts $x \neq y$. To complete the proof, we suppose that

$$
\lim _{s} \inf \|z(s)-y\|<\|z-y\| .
$$

Then, for every $\alpha \in G$, there exist $c>0$ and $t_{\alpha} \in G$ with $t_{\alpha} \succcurlyeq \alpha$ such that

$$
\left\|z\left(t_{\alpha}\right)-y\right\|+c<\|z-y\|
$$

and there exists $\alpha_{0} E G$ such that

$$
r<\|u(\alpha)-y\|+\frac{c}{2}
$$

for every $\alpha \succcurlyeq \alpha_{0}$. Put $z\left(t_{\alpha}\right)=a_{\alpha} u\left(t_{\alpha}\right)+\left(1-a_{\alpha}\right) z$ for every $\alpha$. Then there is $\beta>0$ and $\gamma<1$ such that $\beta \leqq a_{\alpha} \leqq \gamma$ for every $\alpha \succcurlyeq \alpha_{0}$. In fact, if there exists $a_{\alpha}$ such that $\left(1-a_{\alpha}\right) M<\frac{c}{2}$, where $M \geqq \sup _{t \in G}\|u(t)-z\|$ and $M>c$, then,

$$
\begin{aligned}
& \left|\left\|z\left(t_{\alpha}\right)-y\right\|-\left\|u\left(t_{\alpha}\right)-y\right\|\right| \leqq\left\|z\left(t_{\alpha}\right)-u\left(t_{\alpha}\right)\right\| \\
= & \left(1-a_{\alpha}\right)\left\|u\left(t_{\alpha}\right)-z\right\|<\frac{c}{2}
\end{aligned}
$$


and hence $r \leqq\left\|u\left(t_{\alpha}\right)-y\right\|+\frac{c}{2}<\left\|z\left(t_{\alpha}\right)-y\right\|+c<\|z-y\| \leqq r$. This is a contradiction. If there also exists $a_{\alpha}$ such that $a_{\alpha} M<c$, then

$$
\begin{aligned}
& \left|\left\|z\left(t_{\alpha}\right)-y\right\|-\|y-z\|\right| \leqq\left\|z\left(t_{\alpha}\right)-z\right\| \\
= & a_{\alpha}\left\|u\left(t_{\alpha}\right)-z\right\|<c
\end{aligned}
$$

and hence $\|z-y\|<\left\|z\left(t_{\alpha}\right)-y\right\|+c<\|z-y\|$. This is a contradiction. By (2.1) with $y \in C$ and $D=\{\alpha u(t)+(1-\alpha) z: t \in G, 0 \leqq \alpha \leqq 1\}$, (2.2), and Lemma 3.3, there exists $s_{0} \in G$ with $s_{0} \succcurlyeq \alpha_{0}$ such that

$$
\begin{aligned}
& \|y-S(s) v\|<\frac{c}{3}+\|y-v\|, \\
& \|u(s t)-S(s) u(t)\|<\frac{c}{3},
\end{aligned}
$$

and

$$
\|S(s)(\lambda u(t)+(1-\lambda) z)-(\lambda S(s) u(t)+(1-\lambda) z)\|<\frac{c}{3},
$$

for all $s, t \geqslant s_{0}, v \in D$ and $\lambda \in[\beta, \gamma]$. Therefore, for $s \geqslant s_{0}$, since $t_{s_{0}} \geqslant s_{0}$, it follows that

$$
\begin{aligned}
& \left\|z\left(s t_{s_{0}}\right)-y\right\| \leqq\left\|a_{s_{0}} u\left(s t_{s_{0}}\right)+\left(1-a_{s_{0}}\right) z-y\right\| \\
\leqq & a_{s_{0}}\left\|u\left(s t_{s_{0}}\right)-S(s) u\left(t_{s_{0}}\right)\right\|+\left\|S(s) z\left(t_{s_{0}}\right)-\left(a_{s_{0}} S(s) u\left(t_{s_{0}}\right)+\left(1-a_{s_{0}}\right) z\right)\right\| \\
& +\left\|S(s) z\left(t_{s_{0}}\right)-y\right\|<\left\|z\left(t_{s_{0}}\right)-y\right\|+c<\|z-y\| .
\end{aligned}
$$

Let $\beta_{0}=s_{0} t_{s_{0}}$ and $s \geqslant \beta_{0}$. Since $G$ is right reversible, we have

$$
\sup _{s \geq \beta_{0}}\|z(s)-y\|=\sup _{s \geq s_{0}}\left\|z\left(s t_{s_{0}}\right)-y\right\|<\|z-y\| .
$$

Thus, we have $z(s) \neq z$ for all $s \geqslant \beta_{0}$. Now let $s \geqslant \beta_{0}$ and $u_{k}=k(z-z(s))+z(s)$ for all $k \geqq 1$. Then $\left\|u_{k}-y\right\| \geqq\|z-y\|$ for all $k \geqq 1$ and hence, by Theorem 2.5 of $[2]$, we have

$$
\left\langle z-u_{k}, J(y-z)\right\rangle=\langle(1-k)(z-z(s), J(y-z)\rangle \geqq 0
$$

for all $k \geqq 1$, where $J$ is the duality mapping of $E$ and $\left\langle x, x^{*}\right\rangle$ denotes the value of $x^{*} \in X^{*}$ at $x \in E$. Then it follows that $\langle z-z(s), J(y-z)\rangle \leqq 0$ for all $s \geqslant \beta_{0}$. Then, since $z(s) \in[u(s), z]$, this easily implies that $\langle z-u(s), J(y-z)\rangle \leqq 0$ for all $s \geqslant \beta_{0}$. Immediately, we obtain $\langle z-w, J(y-z)\rangle \leqq 0$ for all $w \in \overline{c o}\{u(s)$ : $\left.s \geqslant \beta_{0}\right\}$. Put $w=x=z+(z-y)$, then $z=y$. This contradicts $x \neq y$. The proof is completed.

As a direct consequence, we present the following weak convergence of an almost-orbit $\{u(t): t \in G\}$. 
THEOREM 3.6. Let $G$ be right reversible and let $E$ be uniformly convex with a Fréchet differentıable norm. Suppose that $u=\{u(t): t \in G\}$ is an almost-orbit of $\Im=\{S(t): t \in G\}$ and $F(\Im) \neq \emptyset$. If $\omega_{w}(u) \subseteq F(\Im)$, then the not $\{u(t): t \in G\}$ converges weakly to an element of $F(\Im)$.

Proof. Be similar to Theorem 3 of [15].

\section{Ergodic theorems}

We now study in this section the existence of a "ergodic" retraction of $C$ onto the common fixed point set $F(\Im)$ of $\Im=\{S(t): t \in G\}$ in $C$. We begin with the following observation:

THEOREM 4.1. Let $G$ be right reversible and let $E$ be uniformly convex. Then, the set $F(\mathfrak{\Im})$ (possibly empty) is closed and convex.

Proof. By continuity of elements of $\mathfrak{\Im}$, obviously $F(\mathfrak{\Im})$ is closed. To prove the convexity of $F(\mathfrak{\Im})$, it suffices to show that, for $x, y \in F(\mathfrak{\Im})$ with $x \neq y, z=\frac{1}{2}(x+y) \in F(\Im)$. If $\lim _{t} S(t) z=z$, since $G$ is right reversible, we have $S(s) z=\lim _{t} S(s t) z=\lim _{t} S(t) z=z$ for each $s \in G$ and so $z \in F(\Im)$. Hence it suffices to show that $\lim _{t} S(t) z=z$. If not, there exists $\varepsilon>0$ such that for any $\alpha \in G$, there is $t_{\alpha} \in G$ with $t_{\alpha} \geqslant \alpha$ and

$$
4\left\|S\left(t_{\alpha}\right) z-z\right\| \geqq \varepsilon .
$$

Since $E$ is uniformly convex, choose $d>0$ so small

$$
(R+d)\left(1-\delta\left(\frac{\varepsilon}{R+d}\right)\right)<R,
$$

where $R=\|x-y\|>0$ and $\delta$ is the modulus of convexity of $E$.

For this $d>0$, since $\Im$ is of w.a.n.t. with $z \in C$ and $D=\{x, y\}$, there is $\alpha_{0} \in G$ such that, for all $t \geqslant \alpha_{0}$,

$$
\|S(t) z-w\|=\|S(t) z-S(t) w\| \leqq\|z-w\|+\frac{d}{2} \text { for all } w \in D .
$$

Thus, $2\|S(t) z-x\|, 2\|S(t) z-y\| \leqq R+d$ for all $t \geqslant \alpha_{0}$. Put $u=2\left(S\left(t_{\alpha_{0}}\right) z-x\right)$, $v=2\left(y-S\left(t_{\alpha_{0}}\right) z\right)$. Then, $\|u\|,\|v\| \leqq R+d$ and $\|u-v\|=4 \| S\left(\left(t_{\alpha_{0}}\right) z-z\right) \geqq \varepsilon$. So, we have

$$
R=\left\|\frac{u+v}{2}\right\| \leqq(R+d)\left(1-\delta\left(\frac{\varepsilon}{R+d}\right)\right)<R,
$$

which gives a contradiction. This completes the proof.

As a direct consequence, we get the following: 
Corollary 4.2. Let $E$ be uniformly convex. If a mapping $T: C \rightarrow C$ is of weakly asymptotically nonexpansive type, then the fixed point set $F(T)$ of $T$ is in fact closed and convex.

For each $x \in C$, we define $u(t)=S(t) x(t \in G)$. Then $\{u(t): t \in G\}$ is obviously an almost-orbit of $\Im=\{S(t): t \in G\}$. As a direct consequence, we can prove the following result which generalizes Theorem 8 in [11]. We employs the method of the proof in [11].

THEOREM 4.3. Let $G$ be right reversible and let $E$ be uniformly convex with a Fréchet differentiable norm. Let $\mathfrak{\Im}=\{S(t): t \in G\}$ be of w.a.n.t on $C$. The following are equivalent:

(i) $\bigcap_{s \in G} \overline{c o}\{S(t) x: t \geqslant s\} \cap F(\Im) \neq \emptyset$ for each $x \in C$;

(ii) there exists a retraction $P$ of $C$ onto $F(\Im)$ such the $P S(t)=S(t) P=P$ for every $t \in G$ and $P x \in \overline{c o}\{S(t) x: t \in G\}$ for every $x \in C$.

Proof. By Theorem 3.5, for each $x \in C, \bigcap_{s \in G} \overline{c o}\{S(t) x: t \geqslant s] \cap F(\mathfrak{S})$ contains exactly one point $P x$. Then, applying the same method of [11, Theorem 8], (i) implies (ii). The converse implication is easy.

\section{REFERENCES}

[1] W.L. Bynum, A class of spaces lacking normal structure, Compositio Math., 25 (3) (1972), 233-236.

[2] F.R. Deutch and P.H. Maserick, Applications of the Hahn-Banach theorem in approximation theory, Siam Rev., 9(3) (1967), 516-530.

[3] G. Emmanuele, Asymptotic behavior of iterates of nonexpansive mappings in Banach spaces with Opial's condition, Proc. Amer. Math. Soc., 94(1) (1985), 103-109.

[4] K. Goebel and S. Reich, Uniform convexity, hyperbolic geometry, and nonexpansive mappings, Monographs and Textbooks in Pure and Applied Mathematics, 83, Marcel Dekker, Inc., New York and Basel, 1984.

[5] J.P. Gossez and E. Lami Dozo, Some geometric properties related to the fixed point theory for nonexpansive mappings, Pacific J. Math., 40(3) (1972), 565-573.

[6] C.W. Groetsh, A note on segmenting Mann iterates, J. Math. Anal. Appl., 40 (1972), 369-372.

[7] R.D. Holmes and A.T. LAU, Nonexpansive actions of topological semigroups and fixed points, J. London Math. Soc., (2) 5 (1972), 330-336.

[8] M.T. KIANG and K.K. TAN, Fixed point theorem for proximately nonexpansive semigroups, Canad. Math. Bull., 29(2) (1986), 160-166.

[9] W.A. KIRK, Fixed point theorems for non-Lipschitzian mappings of asymptot1cally nonexpansive type, Israel J. Math., 17 (1974), 339-346.

[10] A.T. LAU, Semigroup of nonexpansive mappings on a Hilbert space, J. Math. Anal. Appl., 105 (1985), 514-522.

[11] A.T. LAU and W. TAKahashi, Weak convergence and non-linear ergodic theorems for reversible semigroups of nonexpansive mappings, Pacific J. Math. 
126 (2) (1987), 277-294.

[12] I. Mryadera, Asymptotic behavior of iteratess of nonexpansive mappings in Banach spaces, Proc. Japan Acad., 54(A) (1978), 212-214.

[13] I. Miyadera and K. Kobayashi, On asymptotic behavior of almost-orbits of nonlinear contraction semigroups in Banach spaces, Nonlinear Analysıs, 6(4) (1982), 349-365.

[14] Z. OpIAL, Weak convergence of the sequence of successive approximations for nonexpansive mappings, Bull. Amer. Math. Soc., 73 (1967), 591-597.

[15] W. TAKahashi and Pei-Jun Zhang, Asymptotic behavior of almost-orbits of semigroups of Lipschizian mappings in Banach spaces, Kodai Math. J. 11 (1988), 129-140.

Department of Applied Matiematics

National Fisheries University of Pusan

Pusan 608-737, Korea 